\title{
Synthesis of zeolites using residues from mining as aluminum source
}

\section{Gabriel B. Báfero*, Heloise O. Pastore}

\begin{abstract}
Zeolites are framework aluminosilicates extensively used in industry. The quest for cheap high-quality chemicals added to the growing concern with the planet's environmental conditions have stimulated continuous development and upgrading of resources for materials syntheses. In this work, zeolites from MFI, LTA and FAU groups, largely applied indystrially, were synthetized from mineral Al-rich residues.
\end{abstract}

\section{Key words:}

Zeolites, Mining residue, Environment.

\section{Introduction}

For many years zeolites have found important applications in several areas, especially as catalysts in the oil refining and petrochemical industry because of their superior activity, stability and selectivity in major conversion processes. Such features are due, in particular, to their unique structures with completely linked frameworks of $\left[\mathrm{SiO}_{4}\right]$ and $\left[\mathrm{AlO}_{4}\right]$ of tetrahedra. The framework contains open spaces in the form of channels and cages. ${ }^{1}$

One of the most requested zeolites in industry is ZSM-5 (MFI), especially used for acid-catalyzed reactions such as hydrocarbon isomerization and the alkylation of hydrocarbons. ${ }^{2}$ Its structure is formed by medium-pores of 10 membered rings. Zeolite Linde type A (LTA) has small-pores of 8 membered rings and zeolite Linde type $X$ (FAU) has large-pores of 12 membered rings. Their structures can be seen in Figure 1.
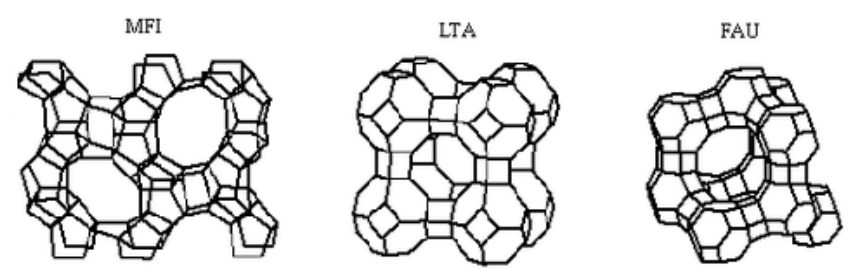

Figure 1. The structure of MFI, LTA and FAU zeolites.

\section{Results and Discussion}

The preparation of these materials followed the standard protocol for zeolites syntheses. To a silicate solution, the industrial residue rich in aluminum was added. The mixture was then homogenized and the reaction gel was brought to the hydrothermal treatment. The residue used is very dark in color. Even so, after washing and drying, the zeolites obtained were presented as bright white powders while the mother-liquor was dark tanned, as shown in Figure 2. This is a strong indication that the impurities present in the residue were transferred to the mother-liquor and eliminated during washing.

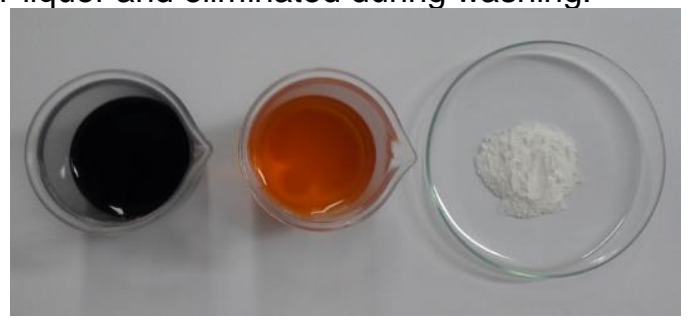

Figure 2. The mining residue, the mother-liquor of the reaction and the zeolite obtained, from left to right.
The materials were analyzed by X-ray diffractometry (XRD) and the profiles obtained are shown in Figure 3. The comparison of these curves with the ones from the literature $^{3}$ shows that no crystalline impurities were produced with the zeolites or impurities from the aluminum source remained in the zeolites prepared from them.

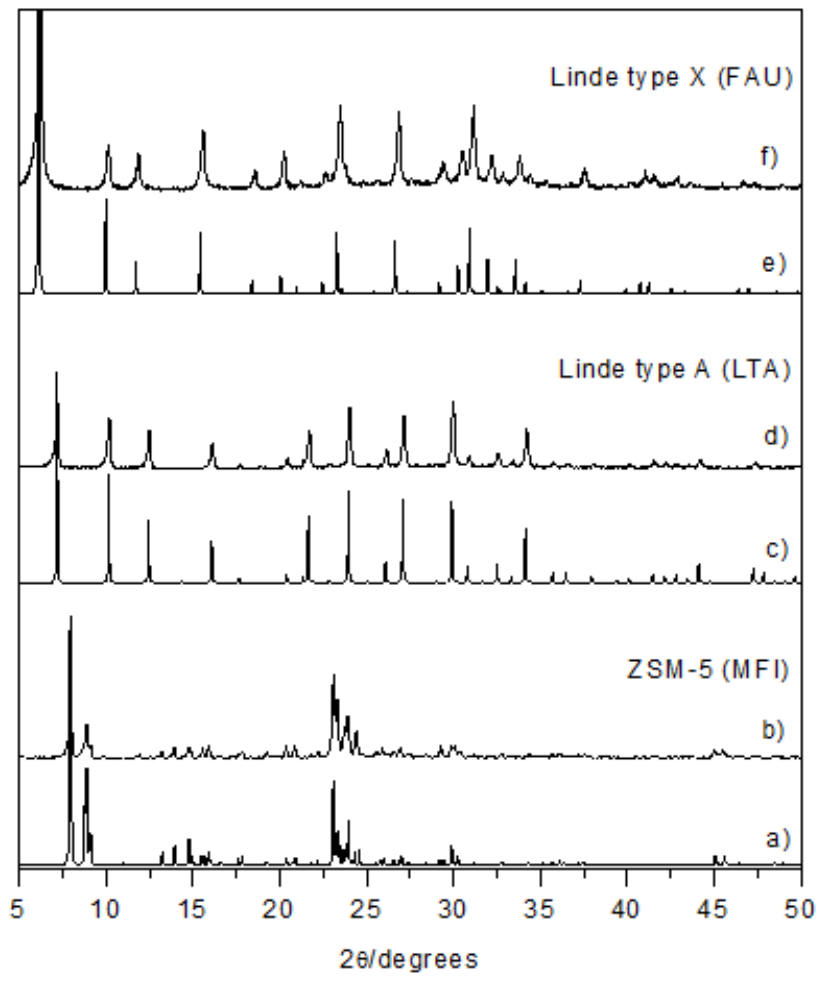

Figure 3. XRD diffractograms from MFI (a,b), LTA (c,d) and FAU (e,f). b, $d$ and $f$ prepared in the lab; $a, c$ and $e$ from the literature.

\section{Conclusions}

The zeolites from MFI, LTA and FAU groups were successfully synthesized through the use of mining residue as aluminum source. The procedures used were of a general type and the products obtained are free from impurities.

\section{Acknowledgement}

Produtos Químicos Guaçu for the financial support. (Convênio 5076)

${ }^{1}$ Davis, M. E. Ind. Eng. Chem. Res. 1991, 30, 1675-1683.

${ }^{2}$ Belussi, G.; Pollesel, P. Stud. Surf. Sci. Catal. 2005, 158, 1201-1212.

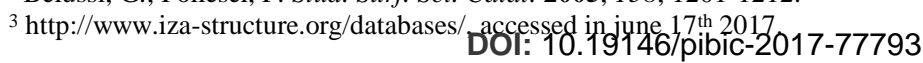

